\title{
A Number Theoretic Approach to Sylow $r$-Subgroups of Classical Groups
}

\author{
Mashhour I. AlAlı, Christoph Hering, \\ and Anni Neumann
Department of Mathematics
Mu'tah University
Alkarak — Jordan \\ masshour_ibrahim@yahoo.com \\ Mathematics Institute \\ University of Tübingen \\ Morgen Stelle 10 \\ D-72076 Tübingen - Germany \\ hering@uni-tuebingen.de \\ Mathematics Institute \\ University of Tübingen \\ Morgen Stelle 10 \\ D-72076 Tübingen - Germany \\ drvneuman@web.de
}

Recibido: 20 de Enero de 2003

Aceptado: 28 de Enero de 2005

\begin{abstract}
The purpose of this paper is to give a general and a simple approach to describe the Sylow $r$-subgroups of classical groups.

Key words: Sylow r-subgroups, wreath product.

2000 Mathematics Subject Classification: 20 D15.
\end{abstract}

\section{Introduction}

Let $G$ be a finite classical group over a finite field of characteristic $p$. The Sylow $r$-subgroups of $G$, where $r$ is a prime number, have been given by Weir [5] in the case $r \neq 2, r \neq p$, and by Chevalley [3] and Ree [4] in the case $r=p$. In the later case the normalizers of the Sylow $p$-subgroups were obtained as well. The remaining case $r=2, p \neq 2$ has been investigated by Carter and Fong [2], where the description is not easy to follow. 
The main purpose of this paper is to give a more general and simple approach to describe the Sylow $r$-subgroups of the general linear group $\operatorname{GL}(n, q)$, the symplectic group $\operatorname{Sp}(2 n, q)$ over $\mathrm{GF}(q), q=p^{a}$, and the symmetric group $S_{n}$, using number theoretic techniques, so that general readers simply can read it. Among other results the conditions on $r$ and $G$ forcing the Sylow $r$-subgroups of $\operatorname{GL}(n, q)$ to be maximal nilpotent are given.

Let $V$ be a $n$-dimensional vector space over $\operatorname{GF}(q)$. In the case of $\operatorname{GL}(V)=$ $\operatorname{GL}(n, q)$, if $d$ is a divisor of $n$, we consider the set $\left\{V_{1}, V_{2}, \ldots, V_{m}\right\}$ of $d$-dimensional subspaces such that $V=V_{1} \oplus V_{2} \oplus \cdots \oplus V_{m}$, where $m=n / d$. Then the stabilizer of this set in $\mathrm{GL}(V)$ is obviously a wreath-product $\mathrm{GL}\left(V_{1}\right)$ ? $S_{m}$. Then we show that for any prime $r \neq p$, the number $d$ can be chosen in such a way that this stabilizer contains a Sylow $r$-subgroup. Hence the Sylow $r$-subgroups are of the form $R \imath T_{m} \leq \mathrm{GL}\left(V_{1}\right) \imath S_{M}$, where $R$ is a Sylow $r$-subgroup of GL $\left(V_{1}\right)$ and $T_{m}$ is a Sylow $r$-subgroup of $S_{m}$. From this description the action of the Sylow $r$-subgroups on the underlying vector space are obvious.

The approach for the other classical groups is quite similar. Let $V$ be a vector space endowed with a bilinear, unitary or quadratic form. Then we consider an orthogonal decomposition $V=V_{1} \perp V_{2} \perp \cdots \perp V_{m}$ into non-degenerate subspaces of equal dimension $d$ say. The stabilizer of the set $\left\{V_{1}, V_{2}, \ldots, V_{m}\right\}$ is then obviously isomorphic to $I\left(V_{1}\right) \backslash S_{m}$, where $I\left(V_{1}\right)$ denotes the isometry group of $V_{1}$. Again by choosing $d$ properly we find the Sylow $r$-subgroups are contained in such stabilizer and hence are isomorphic to $R \imath T_{m}$ where $R$ is a Sylow $r$-subgroup of $I\left(V_{1}\right)$ and $T_{m}$ is a Sylow $r$-subgroup of $S_{m}$. Also the action on the underlying vector space can be immediately seen.

\section{Notation and basic definitions}

Let $n$ be an integer, $p$ prime, we denote by $n_{p}$ the $p$ part of $n$. If $G$ is a finite group, then $|G|$ denotes the order of $G$. If $p$ is prime $\mathbb{Z}_{p-1}$ will denote the multiplicative cyclic group $(\mathbb{Z} / p \mathbb{Z})^{*}$ of the finite field $\mathrm{GF}(p)$. If $g \in G, o(g)$ denotes the order of $g$. Throughout the paper $r, p$ are primes, $r \neq p$, and $q=p^{a}$. H $2 K$ denotes the wreath product of $H$ by $K$. For more information about the wreath product see [1]. $[H: K]$ denotes the index of $K$ in $H$. We write $X^{m}$ for a direct product of $m$ copies of $X$.

\section{The Sylow $r$-subgroups of $\operatorname{GL}(n, q)$}

To investigate the Sylow $r$-subgroups of $\operatorname{GL}(n, q)$, we prove the following Lemmata which are of fundamental importance in this investigation.

Lemma 2.1. Let $d$ be the order of $q+r \mathbb{Z} \in(\mathbb{Z} / r \mathbb{Z})^{*}$, then $\left(q^{i}-1\right)_{r} \neq 1$ if and only if $d \mid i$. 
Proof. Since $|\operatorname{GL}(n, q)|=q^{\left(\begin{array}{c}n \\ 2\end{array}\right)} \prod_{i=1}^{n}\left(q^{i}-1\right)$, we have $|\operatorname{GL}(n, q)|_{r}=\prod_{i=1}^{n}\left(q^{i}-1\right)_{r}$. It is clear that $r \mid q^{i}-1$ if and only if $(q+r \mathbb{Z})^{i}=1+r \mathbb{Z}$, and $(q+r \mathbb{Z})^{i}=1+r \mathbb{Z}$ iff $d \mid i$. Hence the Lemma is proved.

Lemma 2.2. If $r \mid q-1$, then the following properties hold:

(i) If $r \neq 2$, then $\left(q^{i}-1\right)_{r}=i_{r}(q-1)_{r}$.

(ii) If $r=2$ and $q \equiv 1(\bmod 4)$, then $\left(q^{i}-1\right)_{2}=i_{2}(q-1)_{2}$.

(iii) If $r=2$, and $q \equiv 3(\bmod 4)$, then

$$
\left(q^{i}-1\right)_{2}= \begin{cases}2, & \text { if } i \text { is odd } \\ i_{2}(q+1)_{2} & \text { if } i \text { is even }\end{cases}
$$

Proof. Since $r \mid q-1$ we write $q=1+r^{a} x$ for $a \geq 1$ and $\operatorname{gcd}(r, x)=1$. On the other hand, $q^{i}-1=(q-1)\left(1+q+\cdots+q^{i-1}\right)$ implies $\left(q^{i}-1\right)_{r}=(q-1)_{r}\left(1+q+\cdots+q^{i-1}\right)_{r}$. Since $q \equiv 1(\bmod r)$ then $1+q+\cdots+q^{i-1} \equiv i(\bmod r)$.

(i) Case 1: $r \nmid i$. Then $\left(1+q+\cdots+q^{i-1}\right)_{r}=1$ and we are done.

Case 2: $r \mid i$. So $i=r^{b} j$ with $\operatorname{gcd}(j, r)=1$. We need to prove that $(1+q+\cdots+$ $\left.q^{i-1}\right)_{r}=r^{b}$.

Since $q^{i}-1=q^{r^{b} j}-1=\left(q^{j}-1\right)\left(q^{j\left(r^{b}-1\right)}+\cdots+q^{2 j}+q^{j}+1\right)$ then $\left(q^{i}-1\right)_{r}=$ $\left(q^{j}-1\right)_{r}\left(q^{j\left(r^{b}-1\right)}+\cdots+q^{j}+1\right)_{r}=(q-1)_{r}\left(q^{j\left(r^{b}-1\right)}+\cdots+q^{j}+1\right)_{r}$, by case 1. We have also $q^{j\left(r^{b}-k\right)}=\left(1+r^{a} x\right)^{j\left(r^{b}-k\right)} \equiv 1+j\left(r^{b}-k\right) r^{q} x\left(\bmod r^{2 a}\right)$. Thus,

$$
1+\sum_{k=1}^{r^{b}-1} q^{j\left(r^{b}-k\right)} \equiv r^{b}\left(1+j r^{a} x\left(r^{b}-1\right)-j r^{a} x \frac{r^{b}-1}{2}\right) \quad\left(\bmod r^{2 a}\right)
$$

because $r \neq 2$. Therefore

$$
\left(1+q^{j}+\cdots+q^{j\left(r^{b}-1\right)}\right)_{r}=r^{b} .
$$

(ii) We consider the following two cases:

Case 1: $i$ is odd. Then $1+q+\cdots+q^{i-1}$ is odd, and this implies $\left(q^{i}-1\right)_{2}=$ $(q-1)_{2}\left(1+q+\cdots+q^{i-1}\right)_{2}=(q-1)_{2}=i_{2}(q-1)_{2}$.

Case 2: $i$ is even. So $i=2 j$ and $\left(q^{i}-1\right)_{2}=\left(q^{2 j}-1\right)_{2}=\left(q^{j}-1\right)_{2}\left(q^{j}+1\right)_{2}$. Since $q \equiv 1(\bmod 4)$ this implies $q^{j}+1 \equiv 2(\bmod 4)$. Hence $\left(q^{i}-1\right)_{2}=\left(q^{j}-1\right)_{2} \cdot 2=$ $j_{2}(q-1)_{2} \cdot 2=i_{2}(q-1)_{2}$ by induction.

(iii) Again we have two cases: 
Case 1: $i$ is odd. Then $\left(q^{i}-1\right)_{2}=(q-1)_{2}\left(1+q+\cdots+q^{i-1}\right)_{2}=(q-1)_{2}=2$.

Case 2: $i$ is even. So $i=2 j$ and since $q^{2} \equiv 1(\bmod 4)$, then by (ii) we have $\left(q^{i}-1\right)_{2}=$ $\left(q^{2 j}-1\right)_{2}=j_{2}\left(q^{2}-1\right)_{2}=j_{2}(q-1)_{2}(q+1)_{2}=j_{2} \cdot 2 \cdot(q+1)_{2}=i_{2}(q+1)_{2}$.

Lemma 2.3. Let $r$ and $p$ be distinct primes, $q=p^{a}$, and $d=o(q+r \mathbb{Z})$ where $q+r \mathbb{Z} \in(\mathbb{Z} / r \mathbb{Z})^{*}$ then the following properties hold:

(i) If either $r \neq 2$ or $r=2$ and $q \equiv 1(\bmod 4)$, then $|\mathrm{GL}(n, q)|_{r}=\left(q^{d}-1\right)_{r}^{\left[\frac{n}{r}\right]}\left(\left[\frac{n}{d}\right] !\right)_{r}$.

(ii) If $r=2, q \equiv 3(\bmod 4)$ and $n$ is even, then $|\mathrm{GL}(n, q)|_{r}=\left(2^{2}(q+1)_{2}\right)^{\frac{n}{2}}((n / 2) !)_{2}$.

(iii) If $r=2, q \equiv 3(\bmod 4)$ and $n$ is odd, then

$$
|\mathrm{GL}(n, q)|_{r}=2\left(2^{n-1} \prod_{\substack{i \leq n \\ i \text { even }}} i_{2}(q+1)_{2}\right) .
$$

Proof. (i) We have

$$
|\mathrm{GL}(n, q)|_{r}=\left|q^{\left(\begin{array}{c}
n \\
2
\end{array}\right)} \prod_{i=1}^{n}\left(q^{i}-1\right)\right|_{r}=\prod_{i=1}^{n}\left(q^{i}-1\right)_{r}=\prod_{i \leq n, d \mid i}\left(q^{i}-1\right)_{r} .
$$

By Lemma 2.1, $r \mid q^{i}-1$ iff $d \mid i$. We obtain $\prod_{i \leq n, d \mid i}\left(q^{i}-1\right)_{r}=\prod_{j=1}^{\left[\frac{n}{d}\right]}\left(q^{d j}-1\right)_{r}$ and by Lemma 2.2 , with $q$ replaced by $q^{d}$, we obtain

$$
\prod_{j=1}^{\left[\frac{n}{d}\right]}\left(q^{d j}-1\right)_{r}=\prod_{j=1}^{\left[\frac{n}{d}\right]} j_{r}\left(q^{d}-1\right)_{r}=\left(q^{d}-1\right)_{r}^{\left[\frac{n}{d}\right]} \prod_{j=1}^{\left[\frac{n}{d}\right]} j_{r}=\left(q^{d}-1\right)_{r}^{\left[\frac{n}{d}\right]}\left(\left[\frac{n}{d}\right] !\right)_{r} .
$$

Hence a Sylow $r$-subgroup of $\operatorname{GL}(n, q)$ is isomorphic to $Z_{\left(q^{d}-1\right)_{r}} \prec T_{\left[\frac{n}{d}\right]}$, where $T_{\left[\frac{n}{d}\right]}$ is a Sylow $r$-subgroup of $S_{\left[\frac{n}{d}\right]}$.

(ii) $|\operatorname{GL}(n, q)|_{2}=\prod_{i=1}^{n}\left(q^{i}-1\right)_{2}$. Let $n=2 n_{1}$ for some integer $n_{1}$, then we have

$$
\begin{aligned}
\prod_{i=1}^{n}\left(q^{i}-1\right)_{2} & =\prod_{j=0}^{n-1}\left(q^{2 j+1}-1\right)_{2} \prod_{j=1}^{n_{1}}\left(q^{2 j}-1\right)_{2}= \\
& =2^{n / 2} \prod_{j=1}^{n / 2}\left(q^{2 j}-1\right)_{2}=2^{n / 2} \prod_{j=1}^{n / 2}(2 j)_{2}(q+1)_{2}= \\
& =2^{n}(q+1)_{2}^{n / 2} \prod_{j=1}^{n / 2} j_{2}=2^{n}(q+1)_{2}^{n / 2}(n / 2) !=\left(2^{2}(q+1)_{2}\right)^{n / 2}((n / 2) !)_{2}
\end{aligned}
$$


Hence if $n$ is even and $q \equiv 3(\bmod 4)$, then a Sylow 2 -subgroup of $\operatorname{GL}(n, q)$ is isomorphic to $D$ < $T$ where $D$ is a Sylow 2-subgroup of $\operatorname{GL}(2, q)$ and $T$ is a Sylow 2-subgroup of the symmetric group $S_{n / 2}$.

(iii) We have

$$
\begin{aligned}
|\mathrm{GL}(n, q)|_{2} & =\prod_{i=1}^{n}\left(q^{i}-1\right)_{2}=\prod_{\substack{i \leq n \\
i \text { odd }}} 2 \prod_{\substack{i \leq n \\
i \text { even }}}\left(q^{i}-1\right)= \\
& =2^{n} \prod_{\substack{i \leq n \\
i \text { even }}} i_{2}(q+1)_{2}=2 \cdot 2^{n-1} \prod_{\substack{i \leq n \\
i \text { even }}} i_{2}(q+1)_{2} .
\end{aligned}
$$

Hence, if $n$ is odd and $q \equiv 3(\bmod 4)$, then a Sylow 2 -subgroup of $\operatorname{GL}(n, q)$ is isomorphic to $Z_{2} \times S \leq \mathrm{GL}(1, q) \times \mathrm{GL}(n-1, q) \leq \mathrm{GL}(n, q)$, where $S$ is a Sylow 2-subgroup of $\operatorname{GL}(n-1, q)$. The Sylow $r$-subgroups of $S_{n}$ will be discussed in section 4 .

Combining Lemma 2.1 and Lemma 2.2 we have

Lemma 2.4. Let $r$ and $p$ be distinct primes, $q=p^{a}$. Define $d=o(q+r \mathbb{Z})$ where $q+r \mathbb{Z} \in(\mathbb{Z} / r \mathbb{Z})^{*}$, then we have

(i) $r \mid q^{i}-1$ iff $d \mid i$.

(ii) If $d \mid i$ and either $r \neq 2$, or $r=2$ and $q \equiv 1(\bmod 4)$, then $\left(q^{i}-1\right)_{r}=$ $\left(\frac{i}{d}\right)_{r}\left(q^{d}-1\right)_{r}$

(iii) If $d \mid i, r=2$, and $q \equiv 3(\bmod 4)$, then

$$
\left(q^{i}-1\right)_{r}= \begin{cases}2, & \text { if } i \text { is odd } \\ i_{2}(q+1)_{2}, & \text { if } i \text { is even }\end{cases}
$$

Remark 2.5. For $\operatorname{GL}(n, q)$ there are obviously subgroups of the orders calculated above. $\operatorname{GL}(n, q)$ contains the group of the monomial matrices $M \cong Z_{q-1} 2 S_{n}$. So in the case $r \mid q-1, M$ contains a Sylow $r$-subgroup. In general, set $d=o(q+r \mathbb{Z})$ and write $n=n_{0} d+n_{1}$ for integers $n_{0}, n_{1}$ with $0 \leq n_{1}<d$, then we have a canonical embedding of $\operatorname{GL}\left(n_{0} d, q\right)$ into $\operatorname{GL}(n, q)$ as follows. Let $V$ be a vector space of dimension $n$ over $\operatorname{GF}(q)$ and write $V=V_{0} \oplus V_{1}$ where $\operatorname{dim} V_{0}=n_{0} d$, $\operatorname{dim} V_{1}=n_{1}$, so, if $H=\mathrm{GL}\left(V_{1}\right) \times \mathrm{GL}\left(V_{0}\right)$, then $C_{H}\left(V_{0}\right) \cong \mathrm{GL}\left(V_{1}\right)=\operatorname{GL}\left(n_{1}, q\right)$ and $C_{H}(V) \cong$ $\mathrm{GL}\left(V_{0}\right)=\mathrm{GL}\left(n_{0} d, q\right)$. Further, if $W$ is a vector space of dimension $n_{0}$ over $\operatorname{GF}\left(q^{d}\right)$, then $W$ is also a vector space over a subfield $\operatorname{GF}(q) \subseteq \operatorname{GF}\left(q^{d}\right)$ of dimension $n_{0} d$, hence we have a canonical embedding $\mathrm{GL}(W) \subseteq \mathrm{GL}(V)$ or $\operatorname{GL}\left(n_{0}, q^{d}\right) \subseteq \operatorname{GL}\left(n_{0} d, q\right)$. So we get a sequence of embeddings $\operatorname{GL}\left(n_{0}, q^{d}\right) \subseteq \mathrm{GL}\left(n_{0} d, q\right) \subseteq \mathrm{GL}\left(n_{0} d+n_{1}, q\right)=\mathrm{GL}(n, q)$, and $\operatorname{GL}\left(n_{0}, q^{d}\right)$ contains a monomial group $M^{*} \cong Z_{q^{d-1}} 2 S_{n_{0}}$ which contains, as we have shown above, a Sylow $r$-subgroup. 


\section{The Sylow $r$-subgroups of $\operatorname{Sp}(2 n, q)$}

To describe the Sylow $r$-subgroups of $\operatorname{Sp}(2 n, q)$ we prove the following Lemmas.

Lemma 3.1. Let $r$ and $p$ be distinct primes, $q=p^{a}$ and $r$ odd, then

(i) $\operatorname{Sp}(2 n, q)$ contains canonically a subgroup $H$ isomorphic to $\operatorname{GL}(n, q)$.

(ii) If $d$ is odd, then $r$ does not divide the index of $H$ in $\operatorname{Sp}(2 n, q)$, where $d=o(q+\mathbb{Z})$, $q+\mathbb{Z} \in(\mathbb{Z} / r \mathbb{Z})^{*}$.

(iii) Any canonically embedded $\operatorname{GL}(n, q)$ contains a Sylow r-subgroup of $\operatorname{Sp}(2 n, q)$.

Proof. (i) Consider a symplectic base with respect to which the inner product matrix is $\left[\begin{array}{cc}0 & I \\ -I & 0\end{array}\right]$. Then the subgroup

$$
H=\left\{\left[\begin{array}{ll}
g & \\
& \left(g^{t}\right)^{-1}
\end{array}\right], \quad g \in \operatorname{GL}(n, q)\right\}
$$

is contained in the corresponding symplectic group $\operatorname{Sp}(2 n, q)$.

The index of $H$ in $\operatorname{Sp}(2 n, q)$ is

$$
\frac{q^{n^{2}} \prod_{i=1}^{n}\left(q^{2 i}-1\right)}{q^{\left(\begin{array}{c}
n \\
2
\end{array}\right)} \prod_{i=1}^{n}\left(q^{i}-1\right)}=q^{n(n+1) / 2} \prod_{i=1}^{n}\left(q^{i}+1\right) .
$$

(ii) Assume that $r \mid q^{n(n+1) / 2} \prod_{i=1}^{n}\left(q^{i}+1\right)$. This implies that $r \mid q^{i}+1$ for some $1 \leq i \leq n$, so $r \mid q^{2 i}-1$. This means that $q^{2 i} \equiv 1(\bmod r)$, thus $d \mid 2 i$. As $d$ is odd, this implies that $q^{i} \equiv 1(\bmod r)$. Hence $r \mid q^{i}+1$ and $r \mid q^{i}-1$, thus $r \mid 2$, a contradiction.

(iii) As $r \nmid[\operatorname{Sp}(2 n, q): H]$, then $H$ contains a Sylow $r$-subgroup and the Sylow $r$-subgroups of $\operatorname{GL}(n, q)$ have been determined in section 2.

Remark 3.2. If $n=n_{1}+n_{2}$, then $\operatorname{Sp}(2 n, q)$ contains a canonically embedded subgroup $\operatorname{Sp}\left(2 n_{1}, q\right) \times \operatorname{Sp}\left(2 n_{2}, q\right)$. This can be seen as follows. If $V_{1}$ and $V_{2}$ are symplectic spaces, then $V_{1} \oplus V_{2}$ can be turned into a symplectic space, such that $V_{1}$ and $V_{2}$ are orthogonal. Let $\beta_{i}$ be a symplectic form on $V_{i}, i=1,2$. Define a symplectic form $\beta$ on $V_{1} \oplus V_{2}$ by $\beta\left(v_{1}+v_{2}, v_{1}^{\prime}+v_{2}^{\prime}\right)=\beta_{1}\left(v_{1}, v_{1}^{\prime}\right)+\beta_{2}\left(v_{2}, v_{2}^{\prime}\right)$ where $v_{i}, v_{i}^{\prime} \in V_{i}$. At the same time, this defines an embedding of $\operatorname{Sp}\left(V_{1}\right) \times \operatorname{Sp}\left(V_{2}\right)$ into $\operatorname{Sp}\left(V_{1} \perp V_{2}\right)$. Here $V_{1} \perp V_{2}$ denotes that $V_{1}$ and $V_{2}$ are orthogonal by the action

$$
\left(v_{1}, v_{2}\right)^{\left(g_{1}, g_{2}\right)}=\left(v_{1}^{g_{1}}, v_{2}^{g_{2}}\right)
$$

where $v_{1} \in V_{1}, v_{2} \in V_{2}$, and $g \in \operatorname{Sp}\left(V_{1}\right), g_{2} \in \operatorname{Sp}\left(V_{2}\right)$. So we have a canonical embedding $\operatorname{Sp}\left(2 n_{1}, q\right) \times \operatorname{Sp}\left(2 n_{2}, q\right) \subseteq \operatorname{Sp}\left(2\left(n_{1}+n_{2}\right), q\right)$. Repeating this process we get an embedding

$$
\operatorname{Sp}\left(2 n_{1}, q\right) \times \operatorname{Sp}\left(2 n_{2}, q\right) \times \cdots \times \operatorname{Sp}\left(2 n_{k}, q\right) \subseteq \operatorname{Sp}\left(2\left(n_{1}+n_{2}+\cdots+n_{k}\right), q\right),
$$

for any $n_{i} \neq 0$. We have also an embedding $\operatorname{Sp}(2 n, q)^{k} \subseteq \operatorname{Sp}(2 n k, q)$. 
The following Lemma is an immediate consequence of the above remark.

Lemma 3.3. Let $W$ be a symplectic space, and assume that $W$ can be written as an orthogonal direct sum of $V_{1} \perp V_{2} \perp \cdots \perp V_{k}$ of subspaces $V_{i}$ all of the same dimension. Let $H$ be the stabilizer of $\left\{V_{1}, V_{2}, \ldots, V_{k}\right\}$ in $\operatorname{Sp}(W)$, then $H \cong \operatorname{Sp}\left(V_{1}\right) 2 S_{k}$.

Lemma 3.4. Let $r$ and $p$ be distinct primes, $q=p^{a}$. Let $d=o(q+r \mathbb{Z})$ where $q+r \mathbb{Z} \in Z_{r-1}$. If $d$ is even, then

$$
|\operatorname{Sp}(2 n, q)|_{r}=\left(q^{d}-1\right)_{r}^{\left[\frac{2 n}{d}\right]}\left(\left[\frac{2 n}{d}\right] !\right)_{r}
$$

Proof. Let $d=2 t$ for some integer $t$. Then

$$
|\operatorname{Sp}(2 n, q)|_{r}=\prod_{i=1}^{n}\left(q^{2 i}-1\right)_{r}=\prod_{\substack{i=1 \\ d \mid 2 i}}^{n}\left(q^{2} i-1\right)_{r}=\prod_{\substack{i=1 \\ t \mid i}}^{n}\left(q^{2 i}-1\right)_{r}
$$

By setting $i=t j$ we have

$$
\prod_{\substack{i=1 \\ t \mid i}}^{n}\left(q^{2 i-1}\right)_{r}=\prod_{j=1}^{\left[\frac{n}{t}\right]}\left(q^{d j}-1\right)_{r}
$$

By Lemma 2.4, we obtain

$$
\begin{aligned}
\prod_{j=1}^{\left[\frac{n}{t}\right]} j_{r}\left(q^{d}-1\right)_{r} & =\left(q^{d}-1\right)_{r}^{\left[\frac{n}{t}\right]} \prod_{j=1}^{\left[\frac{n}{t}\right]} j_{r}=\left(q^{d}-1\right)_{r}^{\left[\frac{n}{t}\right]}\left(\left[\frac{n}{t}\right] !\right)_{r}= \\
& =\left(q^{d}-1\right)_{r}^{\left[\frac{2 n}{d}\right]}\left(\left[\frac{2 n}{d}\right] !\right)_{r} .
\end{aligned}
$$

Theorem 3.5. Let $r$ be an odd prime, $r \neq p, q=p^{a}$, and $d=o(q+r \mathbb{Z})$. Then the following hold:

(i) If $d$ is odd, then any canonically embedded $\mathrm{GL}(n, q)$ contains a Sylow r-subgroup of $\operatorname{Sp}(2 n, q)$.

(ii) If $d$ is even, $d=2 t$ for $1 \leq t \leq n$ and $n=a t+b$ for $0 \leq b<t$, then any canonically embedded subgroup $\operatorname{Sp}(2 t, q)$ 2 $S_{a} \times \operatorname{Sp}(2 b, q)$ contains a Sylow $r$-subgroup of $\operatorname{Sp}(2 n, q)$ which is isomorphic to $Z_{\left(q^{t}-1\right)_{r}} \imath T$, where $T$ is a Sylow $r$-subgroup of $S_{a}$.

Proof. (i) It follows from Lemma 3.1.

(ii) It is an immediate consequence of Lemma 3.3 and Remark 2.5. 
We are left with the remaining case $r=2$, which will be settled by the following theorem.

Theorem 3.6. The Sylow 2-subgroups of $\operatorname{Sp}(2 n, q)$ are $D \backslash T$ where $D$ is a Sylow 2-subgroup of $\operatorname{Sp}(2, q)=\mathrm{SL}(2, q), T$ is a Sylow 2-subgroup of $S_{n}$, and $q$ is odd.

Proof. By Lemma 2.4, we obtain

$$
|\operatorname{Sp}(2 n, q)|_{2}=\prod_{i=1}^{n}\left(q^{2 i}-1\right)_{2}=\prod_{i=1}^{n} i_{2}\left(q^{2}-1\right)_{2}=\left(q^{2}-1\right)_{2}^{n}(n !)_{2} .
$$

So we have an orthogonal decomposition subgroup $\operatorname{Sp}(2, q)$ l $S n \leq \operatorname{Sp}(2 n, q)$. Hence the Sylow 2-subgroups of $\operatorname{Sp}(2 n, q)$ are as in the Theorem.

\section{The Sylow $r$-subgroups of the symmetric group $S_{n}$}

To complete the description of the Sylow $r$-subgroups of $\operatorname{GL}(n, q)$ and $\operatorname{Sp}(2 n, q)$, we investigate the Sylow $r$-subgroups of $S_{n}$. The following results are useful.

Lemma 4.1. Let $r$ and $p$ be different primes. If $n=p m+r, 0 \leq r<p$. Then $(n !)_{p}=p^{m}\left(\left[\frac{n}{p}\right] !\right)_{p}$.

Proof. We have the identities

$$
(n !)_{p}=\prod_{i=1}^{n} i_{p}=\prod_{j=1}^{\left[\frac{n}{p}\right]}(j p)_{p}=\prod_{j=1}^{\left[\frac{n}{p}\right]} p j_{p}=p^{\left[\frac{n}{p}\right]} \prod_{j=1}^{\left[\frac{n}{p}\right]} j_{p}=p^{\left[\frac{n}{p}\right]}\left(\left[\frac{n}{p}\right] !\right)_{p} .
$$

Corollary 4.2. A Sylow p-subgroup of $S_{n}$ is isomorphic to $Z_{p} \prec T$, where $Z_{p}$ is a Sylow p-subgroup of $S_{p}$ and $T$ is a Sylow p-subgroup of $S_{m}$.

Theorem 4.3. If $T_{n}$ is a Sylow p-subgroup of $S_{n}$, then $T_{n}=Z_{p} \prec\left(Z_{p} \prec\left(Z_{p} \prec T_{\left[n / p^{3}\right]}\right)\right)$. (It is a recursive relation.)

Proof. Let $S_{n}$ act on a set $\Omega$ of size $n$. Let $n=p m+r$, where $0 \leq r<p$. Consider a partition of $\Omega$ by the sets $A_{1}, A_{2}, \ldots, A_{m}, \Gamma$, where $\left|A_{i}\right|=p$ and $|\Gamma|=r$. We have that $\Omega=\bigcup_{i=1}^{m} A_{i} \cup \Gamma$ is a disjoint union. The stabilizer of this partition in $S_{n}$ is $H=\left(S_{p} 2 S_{m}\right) \times S_{r}$, which contains a subgroup $S=Z_{p} \imath T$ where $Z_{p}$ is a Sylow $p$-subgroup of $S_{p}$ and $T$ is a Sylow $p$-subgroup of $S_{m}$. By changing the orders we see that if $T_{n}$ is a Sylow $p$-subgroup of $S_{n}$, then $T_{n}=Z_{p} \prec T_{[n / p]}$ where $T_{[n / p]}$ is a Sylow $p$-subgroup of $S_{[n / p]}$, and $T_{[n / p]}=Z_{p}\left\langle T_{[n / p] / p]}=Z_{p}\left\langle T_{\left[n / p^{2}\right]}\right.\right.$. Hence $T_{n}=Z_{p} \prec\left(Z_{p} \prec T_{\left[n / p^{2}\right]}\right)=Z_{p} \prec\left(Z_{p} \prec\left(Z_{p} \prec T_{\left[n / p^{3}\right]}\right)\right)$. It is a recursive relation. 


\section{A question}

What are the conditions on $r$ and $q$ that force the Sylow $r$-subgroups of $\operatorname{GL}(n, q)$ to be maximal nilpotent? To answer this question we prove the following theorem.

Theorem 5.1. Let $r, p$ be two distinct primes, $d=o(q+r \mathbb{Z})$ where $q+r \mathbb{Z} \in(\mathbb{Z} / r \mathbb{Z})^{*}$. Suppose that $n=m d+k, 0 \leq k<d$, and $R$ is a Sylow $r$-subgroup of $\operatorname{GL}(n, q)$. If $R$ is maximal nilpotent, then $n \equiv 0,1(\bmod d)$ and $q^{d}-1=r^{i}$ for some positive integer $i$.

Proof. Let $S$ be a Sylow $r$-subgroup of GL $(d, q)$. By Schur's Lemma $S$ is cyclic and $|S|=\left(q^{d}-1\right)_{r}$. If $R$ is a Sylow $r$-subgroup of $\operatorname{GL}(n, q)$, then $R=S \imath T$ where $T$ is a Sylow $r$-subgroup of $S_{m}$.

In a matrix form,

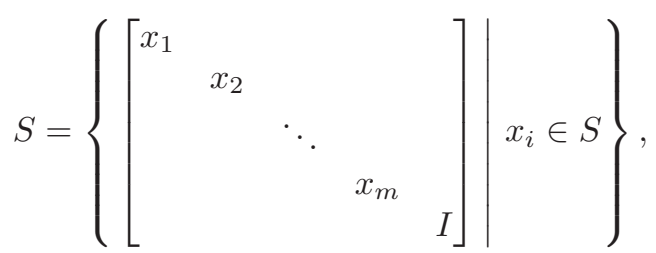

where $x_{i}$ is a $d \times d$ matrix and $I$ is the identity $k \times k$ matrix. Now we prove that $C_{\mathrm{GL}(n, q)}(R)$ is contained in $R$ if $R$ is maximal nilpotent.

Let $x \in C_{\mathrm{GL}(n, q)}(R)$. This implies that $\langle R, x\rangle$ is again nilpotent. Since $R$ is maximal nilpotent, it follows that $R=\langle R, x\rangle$. Thus $x \in R$. It is obvious that all elements

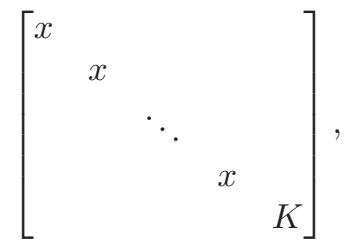

where $K$ is any $k \times k$ matrix and $x \in C_{\mathrm{GL}(d, k)}(S)$, are contained in $C_{\mathrm{GL}(n, q)}(R)$. So if $R$ is maximal nilpotent, all these elements must be contained in $R$. Finally, set

$$
U=\left\{\left[\begin{array}{ccccc}
x & & & & \\
& x & & & \\
& & \ddots & & \\
& & & x & \\
& & & & K
\end{array}\right] \mid x \in C_{\mathrm{GL}(d, q)}(S), \quad K \in \mathrm{GL}(k, q)\right\} .
$$

Then $U \leq C_{G L(n, q)}(R)$. So, if $R$ is maximal nilpotent, this implies $U \leq R$ and hence $U$ must be a $r$-group. Thus $|U|=\left|C_{\mathrm{GL}(d, q)}(S)\right||\mathrm{GL}(k, q)|=\left(q^{d}-1\right)|\mathrm{GL}(k, q)|$ must be a power of $r$. Thus $d^{q}-1=r^{i}$ and $|\mathrm{GL}(k, q)|=r^{j}$, this implies, $k$ must be at most 1 , hence $k=0$ or 1 , which means $n \equiv 0,1(\bmod d)$. 
Acknowledgements. The authors are grateful to Hans J. Schaeffer for his helpful discussions.

\section{References}

[1] M. Aschbacher, Finite group theory, Cambridge Studies in Advanced Mathematics, vol. 10, Cambridge University Press, Cambridge, 1986.

[2] R. Carter and P. Fong, The Sylow 2-subgroups of the finite classical groups, J. Algebra 1 (1964), $139-151$.

[3] C. Chevalley, Sur certains groupes simples, Tôhoku Math. J. (2) 7 (1955), 14-66.

[4] R. Ree, On some simple groups defined by C. Chevalley, Trans. Amer. Math. Soc. 84 (1957), $392-400$.

[5] A. J. Weir, Sylow p-subgroups of the classical groups over finite fields with characteristic prime to $p$, Proc. Amer. Math. Soc. 6 (1955), 529-533. 